\title{
(2) OPEN ACCESS \\ Nationwide inventory on retinopathy of prematurity screening in the Netherlands
}

\author{
Kasia Trzcionkowska (10, ${ }^{1}$ Jacqueline U Termote, ${ }^{2}$ Stefan Böhringer, ${ }^{3}$ \\ Arlette J van Sorge, ${ }^{4}$ Nicoline Schalij-Delfos ${ }^{1}$
}

\begin{abstract}
10phthalmology, Leiden University Medical Center, Leiden, The Netherlands

${ }^{2}$ Neonatology, Wilhelmina Children's Hospital-University Medical Center, Utrecht, The Netherlands

${ }^{3}$ Medical Statistics and Bioinformatics, Leiden University Medical Center, Leiden, The Netherlands

${ }^{4}$ Ophthalmology, Amsterdam UMC Location AMC Amsterdam, The Netherlands
\end{abstract}

\section{Correspondence to} Dr Kasia Trzcionkowska, Ophthalmology, Leiden University Medical Center, Leiden 2333 ZA, The Netherlands;

k.trzcionkowska@lumc.nl

Received 23 June 2021 Accepted 21 November 2021
ABSTRACT

Purpose Provide up-to-date insight in incidence of retinopathy of prematurity (ROP), logistics of screening and treatment in the Netherlands and influence of the new national ROP guideline in which more stringent screening criteria were implemented and the early treatment for ROP criteria (ETROP) were emphasised. Methods Multicentre prospective nationwide study including all preterm infants, born in the Netherlands in 2017, and considered eligible for ROP screening. Anonymised data from ophthalmologists and paediatricians were merged. Outcome data were compared with the first national ROP inventory (NEDROP-1, 2009).

Results In 2017, 1492 infants were live born with gestational age (GA) <32 weeks (2009: 1662); 1287 infants were eligible for screening (2009: 2033). Ophthalmologists screened 1085 infants, versus 1688 in 2009, corrected with factor 1.114 for the difference in number of live births, a $28.4 \%$ (479/1688) decrease in screened infants was seen. Among surviving infants with GA <32 week, ROP was found in 305/1492 babies, 20.4\% (2009: 324/1662, 19.5\%) of which 49/1492 stage $\geq 3,3.3 \%$ (2009: 30/1662, 1.8\%). In all infants, report on presence or absence of plus disease was provided, according to the ETROP criteria. Treatment was performed in 39 infants. Of infants with ROP stage $\geq 3$, $3 / 49(6.1 \%)$ progressed to retinal detachment (2009: 6/30, 20.0\%).

Conclusion The overall ROP incidence expressed as a percentage, remained stable but the number of infants that developed severe ROP nearly doubled. A near onethird reduction in screened infants shows satisfactory implementation of the new screening criteria. A notable decrease in retinal detachment delineates improved treatment outcome.

\section{INTRODUCTION}

Retinopathy of prematurity (ROP) is a mostly selflimiting yet, potentially blinding disease in premature infants. While knowledge and experience on the pathological mechanisms progress, management success rates improve. However, advances in neonatal care lead to increasing survival of extremely premature infants, subsequently causing the group particularly at risk for ROP to grow. ${ }^{1-3}$ Moreover, neonatal risk factors cannot always be fully controlled, therefore, timely screening and treatment remain the foundation for preventing irreversible vision loss due to progressive ROP.

The first Dutch nationwide ROP inventory, the NEDROP study (NEDROP 1, including infants completely screened for ROP and born between 1 January and 31 December 2009), ${ }^{4}$ resulted in the implementation of a new ROP screening and treatment guideline in 2013. ${ }^{56}$

In 2009, ROP was found in 324/1688 (19.2\%) screened infants $(17.4 \%$ mild, $1.8 \%$ severe). Furthermore, critical aspects on the logistics and reporting of screening were revealed: (1) in 624/1688 (37.0\%), first screening was not performed within the required period, (2) risk of loss to follow-up increased with hospital transfer and (3) nearly one-fourth of treated infants (23.5\%) could not be classified according to the early treatment for ROP criteria $\left(\mathrm{ETROP}^{7}\right)$, due to incomplete data reporting on plus disease.

On these findings, several measures were taken. First, quality indicators were added to the Dutch National Monitoring System for Quality in Health Care, in which documentation of the required time period of (follow-up) screening and defined ophthalmological findings in the transferal letter were made obligatory. Second, a parental information folder was developed, which is to be handed before first screening, in order to stress the importance of ROP screening. Third, a section about treatment was added to the guideline, in which the ETROP treatment criteria were emphasised and widely promoted through conferences, courses and so on.

Further, following detailed risk analyses, more stringent and risk factor-based screening inclusion criteria were introduced in $2013,{ }^{6}$ allowing a predicted $29.0 \%$ reduction of screened infants without missing severe ROP. Finally, to help achieve good implementation, a screening and follow-up NEDROP-app was developed for paediatricians and ophthalmologists.

Yet, since 2009, the risk for (severe) ROP increased, due to the lowering of gestational age (GA) limit for active treatment from 25.0 to 24.0 weeks in Dutch neonatal intensive care units (NICUs) $(2010)^{89}$ and implementation of higher oxygen saturation target limits $\left(\mathrm{NeOProM}, 2014^{10}\right)$. As all these policy changes are likely to influence incidence, risk factors and logistics of screening and treatment since the first NEDROP, a second nationwide ROP inventory was performed: the NEDROP 2.

\section{METHODS}

This multicentre, prospective, population-based study was initiated and approved by the Leiden University Medical Center. In the Netherlands, neonatal data are recorded in a national perinatal 
register called Perined. Data are only recorded after parental approval.

All babies born between 1 January and 31 December of the year 2017 who were eligible for ROP screening were reported by neonatologists and paediatricians. They prospectively reported a coded dataset consisting of date of birth, four digits of the ZIP code, GA, birth weight (BW) and the index number in case of multiple birth $(1 / 2,2 / 2,1 / 3$ and so on). Through a separate notification form, ophthalmologists provided the same code of the infants they screened, together with the following ophthalmological findings: date of first examination, suggested and executed dates of follow-up examinations, ROP classification, reason for discontinuation of screening, hospital transfer and, if applicable, date and modality of treatment. Eventually, neonatal and ophthalmological data were merged.

According to the 2013 guideline, ${ }^{11}$ screening applies to infants with GA $<30.0$ weeks and/or BW $<1250 \mathrm{~g}$ and GA 30.0-32.0 weeks and/or BW 1250-1500 g with presence of one or more of the established risk factors: mechanical ventilation, sepsis, necrotising enterocolitis (NEC), postnatal glucocorticoids and hypotension treated with inotropic agents. If the presence of risk factors is uncertain, screening is recommended according to the old guideline, advising examination of all infants with GA $<32.0$ weeks and/or BW < 1500 g. $^{6}$

First screening examination should be scheduled in the 5 th postnatal week (35-42 days), but not before 31.0 weeks of postmenstrual age (PMA). Screening examinations were considered timely if performed within 3 days of scheduled date.

ROP was categorised into type 1 and type 2 ROP. For the purpose of comparison to NEDROP 1, ROP was also classified into mild (stages 1 and 2) or severe (stage 3 or higher, including aggressive posterior ROP) according to the The International Classification of Retinopathy of Prematurityclassification (revisited 2005). ${ }^{12}$

For incidences, national live born premature infants were used as denominator, for which the same cut-off GA was used as in 2009 (<32.0 weeks). Birth rates were obtained from Perined and the Dutch Central Bureau of Statistics. ${ }^{13} 14$

\section{Statistical analysis}

Numerical values are reported as median (25\%-75\% IQR or range). Statistical analysis was performed with SAS Enterprise Guide 7.1 (SAS Institute) and R (V.3.6.1). Frequencies of events were compared using Pearson's $\chi^{2}$ or Fisher's exact test when a cell count was smaller than 5 . For the comparison of multiple groups, pairwise comparisons were calculated. Population parameters were treated as known and a binomial test was used to compare a frequency with a birth rate. Logistic regression was used, when correction for covariates was required.

\section{RESULTS}

\section{Population}

In 2017, 1492 babies with GA $<32.0$ weeks were live born (2009: 1662). Participation of all Dutch hospitals (80) involved in ROP screening was realised, including 10 NICUs, 16 highcare centres (HCs) and 54 regional centres (RCs).

Between 1 January and 31 December of 2017, neonatologists and paediatricians identified 1287 infants eligible for screening (table 1). Infants were born at an NICU (1171; 91.0\%), an HC $(61 ; 4.7 \%)$, RC $(51 ; 4.0 \%)$ and in a foreign (3) or unknown (1) hospital (together 0.3\%). Population characteristics are shown in table $2 \mathrm{~A}$.

\begin{tabular}{|c|c|c|}
\hline & 2009 & 2017 \\
\hline Live born babies & 1662 & 1492 \\
\hline GA $28.0-32.0$ & 1303 & 1092 \\
\hline GA 25.0 to $<28.0$ & 313 & 331 \\
\hline$G A<25.0$ & 46 & 69 \\
\hline Eligible for screening ${ }^{*}$ & 2033 & 1287 \\
\hline Fully screened & $1688(83.0 \%)$ & $1085(84.3 \%)$ \\
\hline Timely first screening & $1064(63 \%)$ & $849(78.2 \%)$ \\
\hline GAt & $30.1(28.6-31.4)$ & $29(27.3-30.4)$ \\
\hline BWt & $1320(1050-1560)$ & $1150(935-1350)$ \\
\hline Infants transferred & 822 & 906 \\
\hline Lost to follow-up after transfer & $189 / 822(23.0 \%)$ & $22 / 902(2.4 \%) \ddagger$ \\
\hline Number of screenings total & 3891 & 3750 \\
\hline Number of screenings no ROP & 2402 & 1981 \\
\hline Number of screenings with ROP & 1489 & 1769 \\
\hline ROP total & 324 & 305 \\
\hline Incidence among live births & $19.5 \%$ & $20.4 \%$ \\
\hline Type 1 ROP§ & 21 & 38 \\
\hline Type 2 ROP§ & 10 & 15 \\
\hline Stage 3 or higher & 30 & 49 \\
\hline Treatment & 17 & 39 \\
\hline Retinal detachment & 6 & 3 \\
\hline
\end{tabular}

*According to Dutch paediatricians.

tMedian (IQR) of screened infants.

¥This group includes only infants who survived and met the new screening criteria §Incomplete data on plus disease for 2009.

$\mathrm{BW}$, birth weight (g); GA, gestational age (weeks); ROP, retinopathy of prematurity.

In total, 1085 babies were fully screened versus 1688 in 2009. Corrected with factor 1.114 for the difference in number of live births between 2017 and 2009 (which gives a hypothetical number of 1209 screened infants), this was a $28.4 \%(479 / 1688)$ reduction of screened infants (table 1).

Two hundred and two infants were not (fully) screened. Of them, 120 died before screening was completed. Fifty-seven infants were wrongly included as did not fit the screening inclusion criteria. The remaining 25 (1.9\% of the infants eligible for screening) were not screened or lost to follow-up because of: transfer abroad (1), no show at follow-up appointment (4) or unknown reason (20).

\section{Screening}

A total of 1085 infants was screened in 3750 screening examinations. Infants who developed ROP were screened 1769 times, those with no ROP, 1981 times (table 1). The number of examinations increased with the severity of ROP (tables 2B and 3).

Data about initial screening and first detection of ROP are shown in table 2B,C. At first screening, 924 infants had no ROP, ROP stage 1 was reported in 125, stage 2 in 34 and stage 3 in 2 infants. In 236 (21.8\%) first screening was performed after the recommended date. At first screening, 40 infants that were screened too late already developed mild (stage 1: 28, stage 2: 12 ) and two stage 3 ROP. Follow-up examinations were carried out timely in $97.1 \%$. In the remaining group, follow-up was performed outside this interval (range 4-67days), without consequences for the outcome.

\section{Retinopathy of prematurity}

Of the 1085 screened infants, 305 (28.1\%) developed ROP. Among 1492 live births with GA <32.0 weeks, the overall ROP incidence was $20.4 \%$, assuming no ROP occurred in the unscreened population (table 1). Median GA and BW were lower in babies developing ROP compared with those that did not 
Table 2 NEDROP 2 population characteristics

\begin{tabular}{|c|c|c|c|c|c|}
\hline & Screened for ROP & No ROP & Overall ROP & Mild ROP & Severe ROP \\
\hline A. The NEDROP 2 study population & 1085 & 780 & 305 & 256 & 49 \\
\hline $\mathrm{GA}^{*}$ & $29.0(27.3-30.4)$ & $29.6(28.3-30.9)$ & $27.1(26.0-28.7)$ & $27.6(26.3-28.9)$ & $25.7(24.9-26.6)$ \\
\hline $\mathrm{BW}^{*}$ & $1150(935-1350)$ & $1213(1029-1414)$ & $938(760-1150)$ & $975(800-1178)$ & $750(319-865)$ \\
\hline Multiple pregnancy, n (\%) & $278(25.6)$ & $192(24.6)$ & $86(28.1)$ & $78(30.5)$ & $8(16.3)$ \\
\hline \multicolumn{6}{|l|}{ B. Screening } \\
\hline Examinations per patient & $3(2-4)$ & $2(1-3)$ & $5(3-7)$ & $4(3-6)$ & $9(8-11)$ \\
\hline First exam PMA & $34.4(32.9-36.0)$ & $35.0(33.6-36.4)$ & $32.6(31.7-34.1)$ & $33.0(31.9-34.3)$ & $31.9(31.2-33.1)$ \\
\hline First exam PNA & $5.4(5.0-6.0)$ & $5.3(5.0-5.9)$ & $5.6(5.0-6.1)$ & $5.4(5.0-6.0)$ & $6.1(5.6-6.9)$ \\
\hline Timely, n (\%) & $849(78.2)$ & $599(76.8)$ & $250(82.0)$ & $211(82.4)$ & $39(79.6)$ \\
\hline Too late, n (\%) & $236(21.8)$ & $181(23.2)$ & $55(18.0)$ & 45 (17.6) & $10(20.4)$ \\
\hline \multicolumn{6}{|l|}{ C. ROP, median (IQR) } \\
\hline First detection PMA & NA & NA & $34.1(33.0-35.7)$ & $34.3(33.0-35.9)$ & $33.6(32.1-34.6)$ \\
\hline First detection PNA & NA & NA & $6.7(5.4-8.3)$ & $6.6(5.3-8.0)$ & $7.3(6.5-9.1)$ \\
\hline
\end{tabular}

Mild ROP: stages $1-2$, severe ROP: stages $\geq 3$

${ }^{*} t$-test no ROP versus overall ROP, $p<0.001$.

BW, birth weight; GA, gestational age; NA, not applicable; PMA, postmenstrual age; PNA, postnatal age; ROP, retinopathy of prematurity.

(both $\mathrm{p}<0.001$, table 2 ). The severity of ROP stages increased with decreasing GA $(p<0.001$, figure 1$)$.

Information on presence or absence of plus disease was provided in 304/305 (99.7\%) of the overall ROP population (present in 41, absent in 263 and not noted in 1 infant with stage 1 ROP), and in $100 \%$ of treated infants. Thirty-eight infants could be categorised into type 1 and 15 into type 2 ROP. For unknown reasons, six babies with type 1 ROP were not treated (ROP3 + inzone II (3) and ROP2+ inzone II (3)). Eventually, in all six, ROP regressed spontaneously. Maximum ROP stage 1 was found in 159 , stage 2 in 97 and stage $\geq 3$ in 49 cases (table 3). At time of first detection, ROP was located in zone I in 22, posterior zone II in 4, zone II in 189 and zone III in 90 cases. Treatment was performed in 39 infants. At treatment decision, 33 had type 1 ROP, 3 had type 2 ROP and 3 could not be categorised because they did not fit the ETROP criteria: two with ROP in zone III and one with stage 2 without plus. Eventually, despite treatment, three babies with ROP stage $3+$ at treatment decision progressed into retinal detachment (tables 1 and 3). Two babies with GA 30.0 weeks—one infant with BW $1415 \mathrm{~g}$, NEC, sepsis and need for inotropic agents for hypotension and one with BW $1360 \mathrm{~g}$, prolonged mechanical ventilation and inhaled nitric oxide-developed stage 3 ROP, illustrating the need for risk-based inclusion criteria to detect outliers in our population.

\section{Transfer}

Overall, 381/1287 (29.6\%) babies were not transferred from the hospital of birth. Other infants were transferred up to six times. In the non-transferred group, the proportion of not screened infants was $126 / 381,33.1 \%$, however, the majority of them died before first screening (116/126, 92.1\%). After excluding the deceased infants and those that were referred according to the old criteria (7), only $3 / 265$ (1.1\%) were not screened. In the transferred group, 76 infants were not screened of which 4 died and 50 were wrongly referred. After exclusion of these, the number of not screened infants was $22 / 902,2.4 \%, \mathrm{p}<0.2$. Logistic regression showed no relation between the number of transfers and number of babies lost to follow-up (OR 1.2, $\mathrm{p}=0.195)$.

\section{DISCUSSION}

The NEDROP 2 study is a prospective, population-based inventory, based on data of all infants born in 2017 and referred for ROP screening in the Netherlands. It is the second national inventory to study the natural course of ROP and adherence to the adapted screening and treatment guideline following the NEDROP 1 (2009). ${ }^{4}$ The then calculated reduction of $29 \%{ }^{6}$ for infants needing ROP screening was found to be $28.4 \%$ in our 2017 study population. However, the number of screening examinations did not decrease as much as the number of screened children (from 3891 in 2009 to 3750 in 2017, a reduction of only $3.6 \%)$. We attribute this to an increase in infants with lower GA $(+18$ with GA $<28.0$ weeks and +23 with GA $<25.0$ weeks), who require relatively more screening examinations. Thus, the implementation of new inclusion criteria for screening in 2013 relieved the burden of screening of the overall population, did not substantially reduce the overall workload for ophthalmologists but enabled them to focus on babies with the highest risk.

Table 3 Characteristics of infants with ROP specified per stage

\begin{tabular}{lllllcc}
\hline & $\mathbf{n}$ & GA & BW & Screenings & Plus disease $(\mathbf{n})$ & $\begin{array}{l}\text { Type 1 } \\
(\mathbf{n})\end{array}$ \\
\hline No ROP & 780 & $29.6(24.0-34.9)$ & $1210(500-2900)$ & $2(1-10)$ & NA & NA \\
ROP 1 & 159 & $28.1(24.4-32.1)$ & $1020(450-2350)$ & $4(1-10)$ & 1 & 0 \\
ROP 2 & 97 & $26.7(24.0-31.4)$ & $898(520-1530)$ & $6(1-17)$ & 9 & 8 \\
ROP 3 & 44 & $25.7(24.0-30.9)$ & $750(410-1415)$ & $10(2-19)$ & 26 & 26 \\
ROP 4 & 2 & $26.0(25.6-26.4)$ & $621(578-665)$ & $14(9-19)$ & 2 & 2 \\
ROP 5 & 1 & 26.1 & 520 & 27 & 1 & 2 \\
APROP & 2 & $25.9(25.1-26.6)$ & $893(785-1000)$ & $7(5-8)$ & 2 & 1 \\
\hline
\end{tabular}

Number of screenings shown as median (minimum-maximum range).

APROP, aggressive posterior ROP; BW, birth weight (g); GA, gestational age (weeks); ROP, retinopathy of prematurity. 


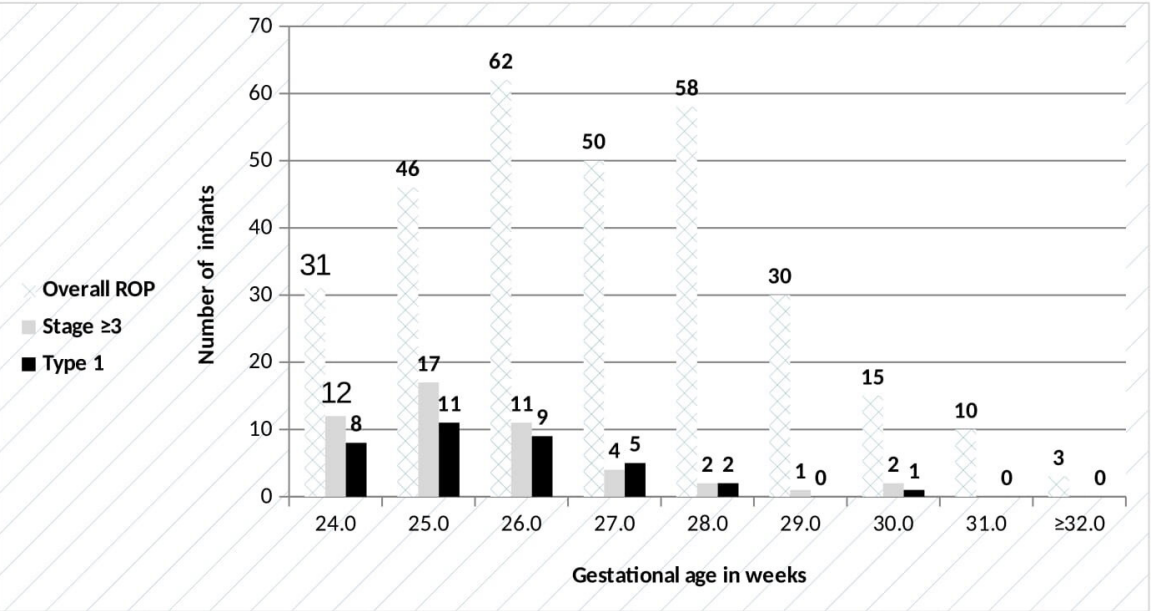

Figure 1 Number of infants with overall, stage $>3$ and type 1 retinopathy of prematurity (ROP) and their distribution of gestational age in weeks. The severity of ROP significantly increased with decreasing GA ( $p<0.001)$. GA, gestational age.

The overall ROP incidence within the screened population increased from $19.2 \%$ in 2009 to $28.1 \%$ in 2017 . However, among all live born babies with GA $<32.0$ weeks, this was $19.5 \%$ in 2009 and $20.4 \%$ in 2017, and thus, relatively stable assuming there was no ROP in the unscreened population (table 1). The increase within the screened population can be therefore attributed to the implementation of risk factor criteria focusing on high risk babies for ROP.

The occurrence of ROP is largely dependent on GA, BW, survival and the presence of ROP associated risk factors during the neonatal period. ${ }^{15}$ Therefore, comparing incidences to other countries is challenging as neonatal policies, survival and screening criteria may differ. Survival for infants born at 24 weeks (as \% of live borns) was 34.4\% in the Netherlands in the period $2011-2017^{16}$. Other similar national population-based cohorts report however survival rates varying from $31 \%$ to $67 \%$ in 24-week infants. ${ }^{17}$ This may be reflected in ROP incidence. Even among cohorts with similar mean population GA and BW, varying ROP incidences can be observed: that is, Sweden (2012: overall $24.0 \%$ of which $8.7 \%$ severe ${ }^{18} ; 2015$ : $24.1 \%$ overall, $8.5 \%$ severe $^{19}$ ), Switzerland (6472 infants between 2006 and 2015: overall $9.2 \%$, severe $1.8 \%{ }^{20}$ ) and a large cohort from 29 Canadian and US hospitals (7483 infants between 2006 and 2011: $43.1 \%$ overall, $6.1 \%$ severe). ${ }^{21}$

We observed an increase in severe ROP (stage $\geq 3$ ) among live born neonates with GA <32.0 weeks, from (30/1662) $1.8 \%$ in 2009 to (49/1492) $3.3 \%$ in 2017 (table 1). Additionally, the absolute number of ROP treatments has more than doubled from 17 (2009) to 39 (2017). This is supported by previous findings, that is, two times as many infants requiring ROP treatment in the Netherlands between 2010 and 2016, found in an earlier retrospective inventory, ${ }^{22} 23$ and findings from countries such as Denmark, ${ }^{24}$ the $\mathrm{UK}^{25}$ and Sweden. ${ }^{3}$ We hypothesise that several essential changes in Dutch neonatal care contributed to this increase.

First, in 2010 the age limit for active neonatal treatment was lowered from 25.0 to 24.0 weeks of gestation. ${ }^{89}$ As anticipated, this has indeed led to a higher number of infants who, based on GA, are at particular risk for severe ROP. While the overall number of preterm infants with GA $<32.0$ weeks decreased from 1662 (2009) to 1492 (2017), the subgroup born GA $<25.0$ weeks increased by $50 \%$ (table 1 ). Although survival of extremely premature infants in the Netherlands is still in the lower range compared with other high income countries, with continuously improving survival of these neonates, awareness of concomitant conditions such as (severe) ROP remains crucial. ${ }^{16}$ Second, surprisingly the NEDROP 1 revealed that the ETROP criteria, ${ }^{7}$ which apply since their publication in 2004, were not yet fully implemented in the Netherlands. ${ }^{4}$ The former guideline dating from 1997 did not include a directive on ROP treatment, therefore possibly, utilisation into practice stayed behind. Subsequently, the criteria were emphasised in the 2013 guideline, which might lead to treatment decision in earlier (less advanced) stages and therefore, might increase the number of infants requiring treatment. The present study shows a notable improvement of report on plus disease in both overall population and treated group (from $83.0 \%$ to $99.7 \%$ and $76.5 \%$ to $100 \%$, respectively), delineating the need for periodic monitoring of screening and treatment outcomes in order to improve national guidelines and their implementation. Although the proportion of infants with end stage ROP decreased, ROP stages at treatment decision were comparable in both studies and thus truly earlier treatment was not observed. So more awareness for signs of disease progression remains important.

Third, following the NeOProM meta-analysis, higher oxygen saturation targets are now globally advised, ${ }^{10} 26$ because of better survival and lower risk of NEC. Simultaneously however, an increased risk for severe ROP is expected. Since in the Netherlands higher $\mathrm{SaO} 2$ target limits were accepted in most NICUs, we hypothesise that when applied during the first weeks of life, the oxygen regime could have contributed to the increase in severe ROP.

ROP is predominantly self-limiting. However, undiagnosed and untreated progressive stages can lead to devastating and lifelong consequences. Our inventory demonstrates the benefits of measures taken to promote timely screening, as this number notably decreased from $624 / 1688,37.0 \%$ in $2009^{4}$ to $236 / 1085,21.8 \%$ $(p<0.001)$. Still, over one-fifth is not screened within the required period. Moreover, two infants already had stage 3 ROP at first screening, performed at 6 and 10 weeks PMA. Therefore, as ROP has a narrow window of opportunity for treatment, the importance of well-timed screening must be stressed continuously.

The well-known obstacle in ROP screening of loss to follow-up due to hospital transfer ${ }^{27} 28$ was addressed in the 2013 ROP guideline and our results illustrate the positive effect of the increased awareness of both physicians and parents: within the transferred group, the number of not screened infants decreased from 189/822, 23.0\% 
$(2009)^{4}$ to $22 / 902,2.4 \%$ (2017). Still, the absolute number of loss to follow-up is two times as high in transferred infants compared to the non-transferred group. Considering a higher number of transferred infants compared to NEDROP 1, marked by the fact that in 2017 only $29.6 \%$ of all infants were fully screened in hospital of birth (vs $59.6 \%$ in 2009), we underline this issue of concern in future hospital transfers.

The main strength of this study is its design which gives insight in annual incidence, screening and treatment of ROP in the Netherlands. Data were provided by both ophthalmologists and paediatricians, therefore apart from nationwide insight in ROP, screening referral and guideline adherence could also be monitored. The identical study design allowed comparison with NEDROP 1 and thus, to evaluate the influence of policy changes in ROP care. Still, due to the new screening inclusion criteria, many infants with a low ROP risk were no longer included in the present study, thus, the two study populations were not entirely comparable. To correct for this difference, the number of surviving infants was used as denominator instead of the screened population. Finally, all data were collected anonymously. For this reason, in infants who were not screened, it was impossible to determine if they eventually developed ROP.

To conclude, the overall ROP incidence in the Netherlands was comparable to 2009 , but the number of infants with treatment requiring ROP nearly doubled. A 28.4\% reduction in infants screened for ROP was accomplished since the implementation of new, risk-based screening inclusion criteria, shifting the focus of ROP screening to babies with the highest risk. The number of infants lost to follow-up due to hospital transfer has decreased, but the risk for not being screened remains. Although the general opinion might be that the screening programme functions properly, this study shows that periodic evaluation is valuable and should be mandatory.

Acknowledgements We thank all participating ophthalmologists, neonatologists and pediatricians for their contribution.

Contributors Guarantor KT. Research design was done by KT, NS-D and JUT. Acquisition and analysis of data were performed by KT and AJvS. The authors SB and KT were responsible for statistical analysis. The article was written by KT, NS-D and JUT and was critically revised and approved for publication by AJvS.

Funding This study was supported by a grant from ODAS Foundation, Delft, the Netherlands. Data were presented at the Association for Research in Vision and Ophthalmology (ARVO) and European Paediatric Ophthalmological Society (EPOS) annual meetings in 2019 for which at both meetings a Travel Grant was rewarded.

Competing interests None declared.

Patient consent for publication Not applicable.

Ethics approval This study does not involve human participants.

Provenance and peer review Not commissioned; externally peer reviewed.

Data availability statement Data are available upon reasonable request. All data are available upon request.

Open access This is an open access article distributed in accordance with the Creative Commons Attribution Non Commercial (CC BY-NC 4.0) license, which permits others to distribute, remix, adapt, build upon this work non-commercially, and license their derivative works on different terms, provided the original work is properly cited, appropriate credit is given, any changes made indicated, and the use is non-commercial. See: http://creativecommons.org/licenses/by-nc/4.0/.

\section{ORCID iD}

Kasia Trzcionkowska http://orcid.org/0000-0003-0666-6746

\section{REFERENCES}

1 Ludwig CA, Chen TA, Hernandez-Boussard T, et al. The epidemiology of retinopathy of prematurity in the United States. Ophthalmic Surg Lasers Imaging Retina 2017:48:553-62.
2 Painter SL, Wilkinson AR, Desai P, et al. Incidence and treatment of retinopathy of prematurity in England between 1990 and 2011: database study. Br J Ophthalmol 2015;99:807-11.

3 Holmström G, Tornqvist K, Al-Hawasi A, et al. Increased frequency of retinopathy of prematurity over the last decade and significant regional differences. Acta Ophthalmol 2018:96:142-8.

4 van Sorge AJ, Termote JUM, Simonsz HJ, et al. Outcome and quality of screening in a nationwide survey on retinopathy of prematurity in the Netherlands. Br I Ophthalmol 2014;98:1056-60.

5 van Sorge AJ, Termote JUM, Kerkhoff FT, et al. Nationwide inventory of risk factors for retinopathy of prematurity in the Netherlands. J Pediatr 2014;164:494-8.

6 van Sorge AJ, Schalij-Delfos NE, Kerkhoff FT, et al. Reduction in screening for retinopathy of prematurity through risk factor adjusted inclusion criteria. $\mathrm{Br} J$ Ophthalmol 2013:97:1143-7.

7 Good WV, Early Treatment for Retinopathy of Prematurity Cooperative Group. Final results of the early treatment for retinopathy of prematurity (ETROP) randomized trial. Trans Am Ophthalmol Soc 2004;102:233-48.

8 de Laat MWM, Wiegerinck MM, Walther FJ, et al. [Practice guideline 'Perinatal management of extremely preterm delivery']. Ned Tijdschr Geneeskd 2010;154:A2701.

9 de Kluiver $\mathrm{E}$, Offringa $\mathrm{M}$, Walther FJ, et al. [Perinatal policy in cases of extreme prematurity; an investigation into the implementation of the guidelines]. Ned Tijdschr Geneeskd 2013;157:A6362.

10 Saugstad OD, Aune D. Optimal oxygenation of extremely low birth weight infants: a meta-analysis and systematic review of the oxygen saturation target studies. Neonatology 2014;105:55-63.

11 Dutch retinopathy of prematurity guideline. Available: https://richtlijnendatabase.nl/ richtlijn/prematuren_retinopathie/screeningsschema_rop.html

12 International Committee for the Classification of Retinopathy of Prematurity. The International classification of retinopathy of prematurity revisited. Arch Ophthalmol 2005:123:991-9.

13 Perined http://www. perined.nl

14 Central Bureau of statistics (CBS) https://www.cbs.nl/

15 Hellström A, Smith LEH, Dammann O. Retinopathy of prematurity. Lancet 2013:382:1445-57.

16 van Beek PE, Groenendaal F, Broeders L, et al. Survival and causes of death in extremely preterm infants in the Netherlands. Arch Dis Child Fetal Neonatal Ed 2021;106:251-7

17 Patel RM, Rysavy MA, Bell EF, et al. Survival of infants born at Periviable gestational ages. Clin Perinatol 2017;44:287-303.

18 Holmström GE, Hellström A, Jakobsson PG, et al. Swedish national register for retinopathy of prematurity (SWEDROP) and the evaluation of screening in Sweden. Arch Ophthalmol 2012;130:1418-24.

19 Holmström G, Hellström A, Jakobsson P, et al. Evaluation of new guidelines for ROP screening in Sweden using SWEDROP - a national quality register. Acta Ophthalmol 2015;93:265-8.

20 Gerull R, Brauer V, Bassler D, et al. Incidence of retinopathy of prematurity (ROP) and ROP treatment in Switzerland 2006-2015: a population-based analysis. Arch Dis Child Fetal Neonatal Ed 2018;103:F337-42.

21 Quinn GE, Ying G-S, Bell EF, et al. Incidence and early course of retinopathy of prematurity: secondary analysis of the postnatal growth and retinopathy of prematurity (G-ROP) study. JAMA Ophthalmol 2018;136:1383-9.

22 Trzcionkowska K, Vehmeijer WBHJ, van Rijn LJ, et al. Increase in treatment of severe retinopathy of prematurity following a new national guideline. Acta Ophthalmol 2018;96:e1033-4.

23 Trzcionkowska K, Vehmeijer WBHJ, Kerkhoff FT, et al. Increase in treatment of retinopathy of prematurity in the Netherlands from 2010 to 2017. Acta Ophthalmol 2021:99:97-103.

24 Slidsborg C, Olesen HB, Jensen PK, et al. Treatment for retinopathy of prematurity in Denmark in a ten-year period (1996 2005): is the incidence increasing? Pediatrics 2008;121:97-105

25 Tavassoli S, Wach R, Haynes R, et al. Estimate of incidence of ROP requiring treatment in extreme preterms and impact on service -7 year review in tertiary unit. Eye 2019:33:845-9.

26 Sweet DG, Carnielli V, Greisen G, et al. European Consensus Guidelines on the Management of Respiratory Distress Syndrome - 2016 Update. Neonatology 2017:111:107-25.

27 Wiggins RE, Gold RS, Menke AM. Twenty-Five years of professional liability in pediatric ophthalmology and strabismus: the OMIC experience. J Aapos 2015; 19:535-40.

28 Aprahamian AD, Coats DK, Paysse EA, et al. Compliance with outpatient followup recommendations for infants at risk for retinopathy of prematurity. J Aapos 2000:4:282-6 